\title{
NUMERICAL MODELING OF FERRITE MATERIAL IMPACT ON THE EFFECTIVENESS OF INDUCTION HEATING PROCESS
}

The article deals with numerical simulation of induction heating process as a coupling of electromagnetism and heat transfer. The article presents how the surrounding materials used in the induction heating process influence the resulting temperature. The scope of the article is to show impact of ferrite material on the resulting temperature of induction heating process.

Keywords: Induction heating, finite element method, electromagnetic field analysis, thermal analysis, Joule heat, Maxwell's equations.

\section{Introduction}

Induction heating has been used in industry over the past three decades. Due to its rapid heating and good reproducibility it is used in heat treatment as well as in applications for special use in mechanical engineering and chemical industry. Induction heating is a non-contact method of heating an electrically conductive material by the process of electromagnetic induction [1]. In a thin layer on the surface of parts alternating electromagnetic field generates currents which are called eddy currents [2]. Eddy currents generate heat due to resistive power losses, which are the main source of heat in the process of induction heating. The induction heating process is dependent on the electrical conductivity of the material, the size of the current in the inductor, the frequency of the applied electromagnetic field and the magnetic properties of the material [3]. Numerical modeling of induction heating allows the optimization of process variables. Using the finite element method, it is possible to calculate the temperature distribution across all components which are exposed to the induction heat. It is thus possible to optimize various process parameters. Induction heating can be addressed as coupling of the electromagnetic and thermal problem [1].

In the following chapters are summarized the fundamental equations and their simplification which are used to describe the physical behavior of materials under electromagnetic and thermal fields.

\section{Electromagnetism equations}

An electromagnetic field describes a set of equations known as Maxwell's equations. Equations consist of Faraday's law and Ampere's law with Maxwell extension.

\footnotetext{
* Andrej Gasparec, Milan Saga, Peter Pechac

Faculty of Mechanical Engineering, University of Zilina, Slovakia

E-mail: milan.saga@fstroj.uniza.sk
}

The equations contain four different variables, the intensity of the electric field $\boldsymbol{E}$, magnetic induction $\boldsymbol{B}$, the intensity of the magnetic field $\boldsymbol{H}$ and the electric induction $\boldsymbol{D}$ [4].

Maxwell's equations are expressed as follows [5 and 4]:

$$
\begin{aligned}
& \nabla \times E=-\frac{\partial B}{\partial t}, \\
& \nabla \times H=J+\frac{\partial D}{\partial t}, \\
& \nabla \cdot B=0, \\
& \nabla \cdot D=\rho .
\end{aligned}
$$

where $J$ is the current density, $t$ is the time and $\rho$ is the free volume charge density.

Constitutive relations between fields in a homogeneous isotropic environment are expressed as follows [5 and 4]

$B=\mu H$,

$D=\varepsilon E$,

$J=\sigma E$,

where $\mu$ is the permeability, $\varepsilon$ is the permitivity of the dielectric and $\sigma$ is the conductivity of the material.

We assume a harmonious solution to the sinus wave. In addition to these relations there is a vector of magnetic potential $A$ in the following relation to

$B=\nabla \times A$. 
Maxwell's equations can then be written by diffusion equation as follows

$\sigma \frac{\partial A}{\partial t}-\frac{1}{\mu} \nabla^{2} A=J_{s}$,

where is the source current density in the inductor.

The diffusion equation (9) can be expressed in the same form for the electric and the magnetic field. In the case of the magnetic vector potential, it can be used in conjunction with any physically noticeable phenomenon of electromagnetic induction, as well as eddy currents induced voltage, inductor impedance, inductor induction. If the current stream as well as eddy currents are considered to be a sinus, and also time-harmonic, electromagnetic fields may be introduced by the following equation [ 6 and 7]

$j \omega \sigma A-\frac{1}{\mu} \nabla^{2} A=J_{s}$.

Depending on the electrical and magnetic properties, there can be intermittent or continuous electromagnetic fields on each side of a common interface between two different materials.

At the interface between two media, the field must meet the following boundary conditions [4, 8 and 9]:

$\hat{n} \times\left(E_{1}-E_{2}\right)=0$,

$\hat{n} \times\left(D_{1}-D_{2}\right)=\sigma$,

$\hat{n} \times\left(H_{1}-H_{2}\right)=J_{s}$,

$\hat{n} \times\left(B_{1}-B_{2}\right)=0$.

The domain border is given by the normal unit vector and is directed from the surface normal of the surface 2 to surface 1 [10 and 11].

Boundary conditions can also be formulated in words such as:

- Components of the electric field are tangentially continuous through the interface of media 1 and 2 .

- The electric flux component is interrupted through an interface with the size $\rho$ for the electrically conductive medium, $\rho=0$.

- The tangential components of the magnetic field intensity are disjointed via two media with size $\boldsymbol{J}$. In the case of a zero surface current and if the medium has final conductivity, the tangential component is continuous [4 and 5].

- Normal component of magnetic flux density is continuous across the interface of media 1 and 2 .

\section{Heat transfer equations}

The formulation of the problem of the energy balance is based on the first thermodynamics law [10]. When dealing with a heat non-stationary event it is necessary to determine the dependence of temperature and the quantity of heat depending on the time for any point of the body. The first law of thermodynamics, heat transfer is characterized by this dependence, which is described by the following equation [12 and 13]:

$\rho c \frac{\partial T}{\partial t}-\nabla \cdot(k \nabla T)=Q$

where $\rho$ is the density, $c$ is the specific heat capacity, thermal conductivity $k$, and $\boldsymbol{Q}$ is the heat generated in the material per unit of volume and time. Heat transfer equation (15) specifies the layout of the temperature in the environment as a function of time and space [14 and 15]. In the case that the temperature distribution is known, it is possible to calculate the heat flux (15) according to the equation inside the body or on its surface. The solution of the equation of heat conduction requires initial and boundary conditions. The boundary condition may be prescribed by a known temperature at the border or by prescribing heat flux using the flux or radiation [16].

The condition of the heat flux at the border can be defined by the relationship

$q_{n}=-k \frac{\partial T}{\partial n}$

where $n$ is normal to the surface towards the outside and $\boldsymbol{q}_{n}$ is a constant heat flux defined by

$q_{n}=h\left(T_{s}-T_{\infty}\right)^{\alpha}+\sigma \varepsilon\left[\left(T_{s}\right)^{4}-\left(T_{\infty}\right)^{4}\right]$,

where $h$ is the convection surface heat transfer coefficient, $T_{s}$ is the surface temperature and $T_{\infty}$ is the ambient temperature.

The first part of the boundary condition represents convective heat transfer and the second part of boundary condition (17) represents radiation.

Suppose that the temperature changes on the surface of the material in the sinusoidal mode. Then it can be shown that the amplitude of the changes will be smaller with increasing depth and with a certain time lag, i.e. the phase angle. If the temperature increases, the frequency attenuation and phase angle will increase [6 and 2]. This is the same phenomenon as in the case of electromagnetic waves, but in a different time scale because the electromagnetic field has a much shorter relaxation time.

In spite of the fact that the diffusion equation (10) has the same properties as heat conduction equation (15), their scales are different. Harmonic current in the device for induction heating is usually applied in the frequency range $50 \mathrm{~Hz}-70$ $\mathrm{kHz}$. For this reason, the use of equation (10) can increase the thermal analysis at each time because the time scale of the electromagnetic problem is much shorter. This assumption is used in the numerical simulation of the solutions strategy [6 and 2]. 


\section{Coupling of electromagnetic and thermal problem}

The practical problem associated with the heat transfer is not usually possible to solve analytically, and especially if it has temperature-dependent and non-linear material properties. The current approach to getting a solution for these problems is to use the finite element method. The aim is to calculate temperature field distribution in the body on which it is applied harmoniously through oscillating current in the inductor.

It is possible to calculate magnetic vector potential from the equation (10). Eddy current $J_{e}$ generated in the body is then calculated using the following relation [2, 3 and 9]

$$
J_{e}=-j \omega \sigma A
$$

The source of the heat in the equation of heat conduction (15) is expressed by [3, 9 and 17]

$Q=\frac{1}{2 \sigma}\left|J_{e}\right|^{2}=\frac{1}{2 \sigma} J_{e} \cdot J_{e}^{*}$

where is the complex conjugate of the .

At the external border area the magnetic vector potential is chosen so that it is zero at the interface (Dirichlet condition). From the equation (10) we can see that the magnetic vector potential is a long-term solution, a solution in which the characteristics of the material of the time step used the $t_{n}$. This is due to the fact that the time scale of the electromagnetic problem is much shorter than the thermal problem.

Figure 1 shows the strategy solutions in the time step. The problem is resolved using the material electromagnetic properties in the time step $t_{n}$, The thermal problem is calculated due to eddy currents in a time step $t_{n+1}$

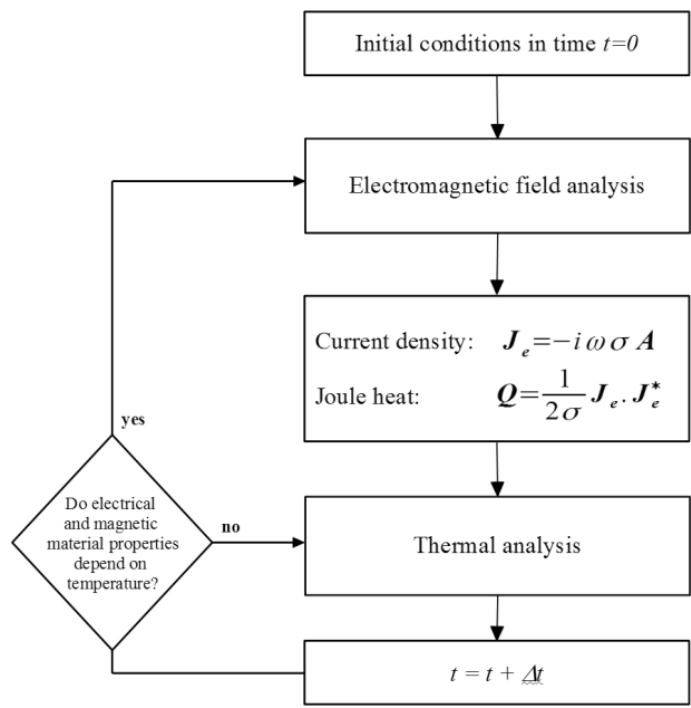

Fig. 1 Solution algorithm of coupling between electromagnetic and heat problem

\section{Two dimensional model of coupled electromagnetic and thermal problem}

Let us assume a general planar n-node element defined in the Cartesian nodal coordinate system $[x, y][18]$.

For a finite n-node element is defined the interpolation of all calculated values in any point inside the element using values from the individual nodal points applying shape functions $N_{i}(x, y)$. For example, the temperature $T(x, y)$ in any point of element can be calculated by the following expression from element nodal temperatures $T_{i}$ [13, 15 and 19]

$T(x, y)=\sum_{i=1}^{n} T_{i} N_{i}(x, y)$.

Energy balance in the modeling area is dedicated to minimizing the energy functional in each node. This can be achieved by setting the first partial derivation of a functional in each node to zero. Instead of minimizing in each node, it is better to perform at the level of element.

The total energy associated to the entire modeling area is then equal to the sum of the energies of all the elements. As a result of the solution of algebraic equations of parallel system with respect to the magnitude of the magnetic vector potential gives an unknown value in each of the nodes. A set of equations can be written in the form

$\left(V_{e}+j W_{e}\right) a_{e}=g_{e}$,

where $V_{e}$ and $W_{e}$ are local matrices corresponding to finite element, $a_{e}$ is the unknown vector of the magnetic vector potential of the corresponding finite element and $g_{e}$ is the vector of the source current density of the corresponding finite element.

The other unknown quantities of the electromagnetic field are obtained using the following expressions [1, 3 and 9].

The intensity of the electric field $E_{z}$

$E_{z}=-j \omega A_{z}$

Electric induction $D_{z}$

$D_{z}=\varepsilon_{0} \varepsilon_{r} E_{z}=-j \omega \varepsilon_{0} \varepsilon_{r} A_{z}$,

where is the relative permittivity and is the vacuum permittivity.

Current density $J_{z}$

$J_{z}=\sigma E_{z}+j \omega D_{z}=-j \sigma \omega A_{z}+\omega \varepsilon_{0} \varepsilon_{r} A_{z}$

Joule heat $Q_{A V}$

$Q_{A V}=\frac{1}{2} \operatorname{real}\left(J_{z} \cdot \dot{E}_{z}\right)$, 
where $\dot{E}_{z}$ is transposed and conjugated (change the sign of the imaginary part of the complex numbers, all the opposite of) the vector of nodal values of electric field intensity $E_{z}$.

By the thermal transient analysis it is possible to solve the system of equations for different time steps, which can be written as follows:

$C_{e} \frac{\partial T}{\partial t}+K_{e} T_{e}=f_{e}$.

Individual matrices and vectors of this system are defined by the following relations:

$C_{e}$, the specific heat matrix defined by

$C_{e}=\int_{\Omega} \rho c_{p} N^{T} N d \Omega=t S \rho c_{p} N^{T} N$.

$K_{e}$ is the heat conductivity matrix defined by expression

$K=\int_{\Omega} B^{T} D B \Omega+\int_{\Gamma} h N^{T} N d \Gamma$.

$D$ is the heat conduction coefficient matrix defined as

$D=\left[\begin{array}{cc}k_{x} & 0 \\ 0 & k_{y}\end{array}\right]$

where $k_{x}$ and $k_{y}$ are heat conduction coefficients for each direction. $N$ is the matrix of shape functions and $\Omega$ is the matrix of their derivations.

In the above mentioned integrals, the represents the volume of the finite element and the represents the boundary of the finite element.

The first integral of the heat transfer matrix (28) characterizes heat conduction and the second integral characterizes heat convection.

The vector on the right side (26) is defined by

$$
f_{e}=\int_{\Omega} G N^{T} d \Omega-\int_{\Gamma} q N^{T} d \Gamma+\int_{\Gamma} h T_{\infty} N^{T} d \Gamma,
$$

where the first integral is characterized by a heat source in the volume, the second integral is characterized by heat flux through the surface area and third integral is characterized by convection through the surface area [13 and 16].

Transient thermal analysis given by a system of equations (26) can be then rewritten as follows

$$
C T^{n+1}=(C-\Delta t K) T^{n}+\Delta t f^{n} .
$$

The solution of the system of equations (31) in the time step " $n$ " is the nodal temperature in the time step " $n+l$ ".

The system of equations (31) is Euler's explicit time integration solutions method (Forward difference) [15 and 20].

\section{Computational model}

Consider two dimensional problem of electromagnetic heating process of six parallel inductors to steel plate specimen. Martensitic annealed stainless steel is used as material for specimens. The scope of the FE study is to observe how different relative permeability of ferrite core material influences final temperature of heated steel plate specimen. The comparison had been done by the same input parameters shown in Table 1 .

Input parameters

Table 1

\begin{tabular}{|l|l|l|}
\hline Current & 300 & {$[\mathrm{~A}]$} \\
\hline Frequency & 3500 & {$[\mathrm{~Hz}]$} \\
\hline Heating time & 50 & {$[\mathrm{~s}]$} \\
\hline Initial temperature & 293.15 & {$[\mathrm{~K}]$} \\
\hline
\end{tabular}

The chosen configuration for FE study runs is shown in Figs. 2 and 3.

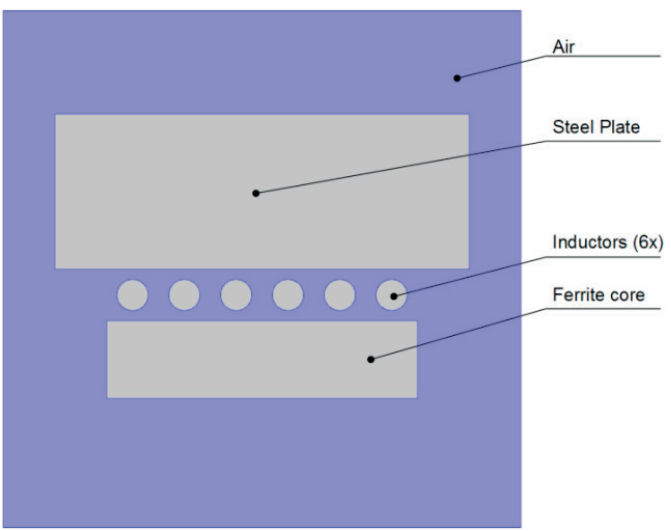

Fig. 2 Computational model

The model size and component dimensions are shown in Fig. 3.

The distances and diameter values are in millimeters $[\mathrm{mm}]$. The length of the model in " $z$ " direction is $100 \mathrm{~mm}$.

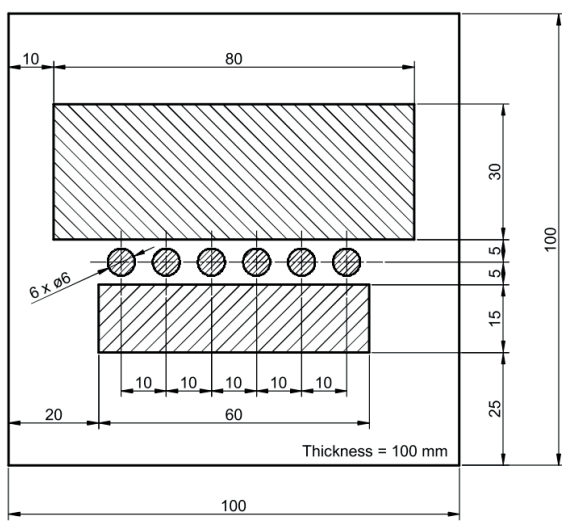

Fig. 3 Model size and component dimensions [mm] 
Free triangular mesh with finer mesh in the areas with the highest value gradients in observed areas was chosen as the FE mesh shown in Fig. 4. The linear 3-node elements are used for interpolation [21].

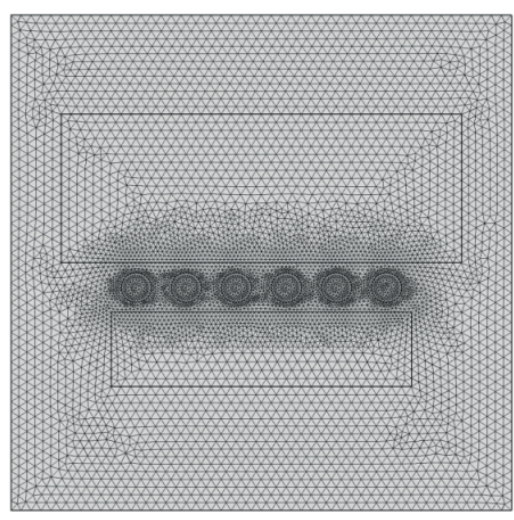

Fig. 4 Finite element mesh

Table 2 shows which material constants were used in FE studies. For the simplification, the constant material properties over the time were considered [22]. The relative permeability of ferrite core marked with $\left(^{*}\right)$ presents the range of the values used in $\mathrm{FE}$ runs.

The inductors were made of copper.

Material properties

Table 2

\begin{tabular}{|l|l|l|l|}
\hline \multirow{4}{*}{ Air } & Material density & 1.293 & {$\left[\mathrm{~kg} / \mathrm{m}^{3}\right]$} \\
\cline { 2 - 4 } & Specific heat & $1.0110^{3}$ & {$[\mathrm{~J} /(\mathrm{kg} \mathrm{K})]$} \\
\cline { 2 - 4 } & Thermal conductivity & $26.010^{-3}$ & {$[\mathrm{~W} /(\mathrm{m} \mathrm{K})]$} \\
\cline { 2 - 4 } & Relative permeability & 1.00000186 & 1 \\
\hline Copper & Material density & 8930 & {$\left[\mathrm{~kg} / \mathrm{m}^{3}\right]$} \\
\cline { 2 - 4 } & Specific heat & 340 & {$[\mathrm{~J} / \mathrm{kg} \mathrm{K}]$} \\
\cline { 2 - 4 } & Thermal conductivity & 384 & {$[\mathrm{~W} /(\mathrm{m} \mathrm{K})]$} \\
\cline { 2 - 4 } & Electrical resistance & $1.710^{-8}$ & {$[\mathrm{ohm} \mathrm{m}]$} \\
\cline { 2 - 4 } & Relative permeability & 0.99999 & 1 \\
\hline Steel & Material density & 7850 & {$\left[\mathrm{~kg} / \mathrm{m}^{3}\right]$} \\
\cline { 2 - 4 } & Specific heat & 460 & {$[\mathrm{~J} /(\mathrm{kg} \mathrm{K})]$} \\
\cline { 2 - 4 } & Thermal conductivity & 28 & {$[\mathrm{~W} /(\mathrm{m} \mathrm{K})]$} \\
\cline { 2 - 4 } & Electrical resistance & $4.3510^{-7}$ & {$[\mathrm{ohm} \mathrm{m}]$} \\
\cline { 2 - 4 } & Relative permeability & 1000 & 1 \\
\hline Ferrite & Material density & 6600 & {$\left[\mathrm{~kg} / \mathrm{m}^{3}\right]$} \\
\cline { 2 - 4 } & Specific heat & 700 & {$[\mathrm{~J} /(\mathrm{kg} \mathrm{K})]$} \\
\cline { 2 - 4 } & Thermal conductivity & 20 & {$[\mathrm{~W} /(\mathrm{m} \mathrm{K})]$} \\
\cline { 2 - 4 } & Electrical resistance & 50000 & {$[\mathrm{ohm} \mathrm{m}]$} \\
\cline { 2 - 4 } & Relative permeability & 1 to $\left.100 \mathrm{(}^{*}\right)$ & 1 \\
\hline
\end{tabular}

\section{Results}

The scope of the numerical model is to calculate resulting temperature after certain heating time. Fig. 5 shows temperature distribution in $[K]$ at $50 \mathrm{~s}$ heating time and used ferrite core relative permeability value of $50\left({ }^{*}\right)$. Fig. 6 presents impact of ferrite material relative permeability resulting temperature of induction heating process.

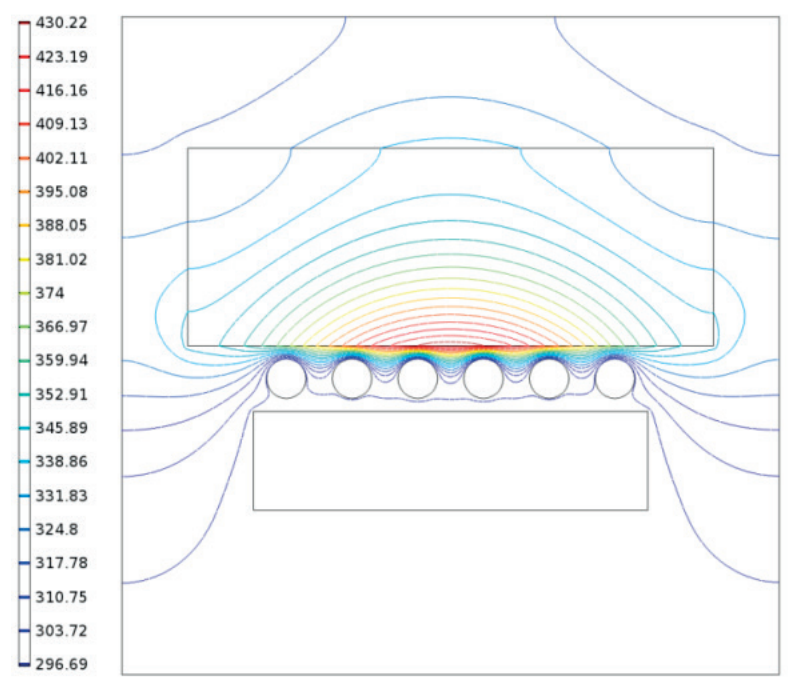

Fig. 5 Resulting temperature distribution at time $50 \mathrm{~s}$ and ferrite core relative permeability value of $50(*)$

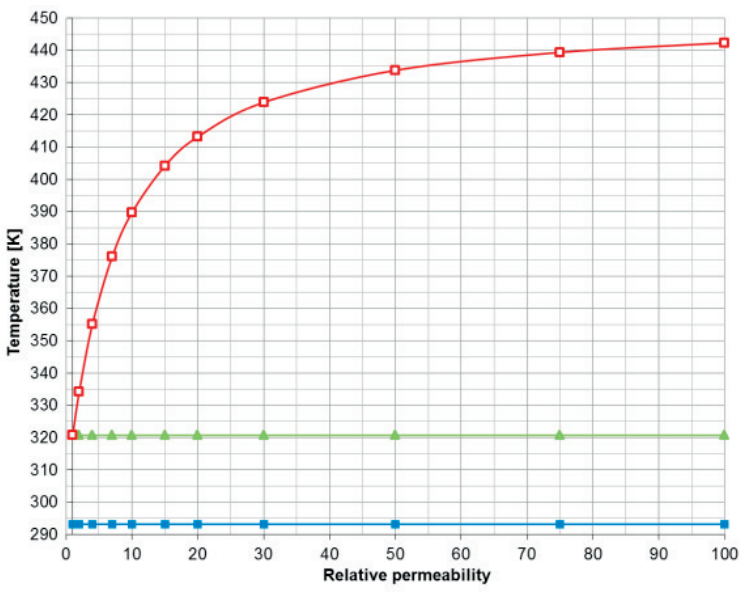

$\rightarrow$-Initial Temperature $\rightarrow-$ Temperature without Ferrite core -0 -Temperature with Ferrite core

Fig. 6 Influence of ferrite core relative permeability (*) on resulting temperature of induction heating process

The red curve in Fig. 6 shows how relative permeability of ferrite core material influences the resulting temperature of heated material. The green line shows the resulting temperature of heated material without ferrite core. Both induction heating processes use the same process settings mentioned in Table 1. 


\section{Conclusion}

In this article we applied equations of the electromagnetic and thermal field to solve problem of induction heating. The numerical simulation of induction heating process is shown as a coupling of electromagnetic and thermal problem leading to a system of thermal transient analysis by the explicit Euler method used in the time integration solutions.

By the FE solution of the electromagnetic and thermal problem it is possible to observe the effect of the individual variables related to electromagnetic induction as well as the resulting temperature or heat generated by the interaction of the surrounding environments for different time steps. By this numerical approach we can observe how different material properties influence the resulting heat generated during the induction heating process and how the right combination of surrounding components influences the heating efficiency.

The numerical modeling of induction heating process allows optimization of the process parameters, material properties of components or geometry shape optimization.

\section{Acknowledgements}

This work has been supported by grant project VEGA No. $1 / 0234 / 13$.

\section{References}

[1] RUDNEV, V., LOVELESS, D., COOK, R., BLACK, M.: Handbook of Induction Heating. Inductoheat, Inc., 2003.

[2] STOLL, R. L.: The Analysis of Eddy Currents. Oxford University Press : Oxford, 1974.

[3] CLAIN, S., RAPPAZ, J., SWIERKOSZ, J., TOUZANI, R.: Numerical Modeling of Induction Heating for Two-dimensional Geometries. Mathematical Models and Methods in Applied Sciences, 3(6), 805-822, 1993.

[4] STRATTON, J. A.: Electromagnetic Theory. IEEE Press : Piscataway, 2007.

[5] KRISTENSSON, G.: Elektromagnetisk vagutbredning. Studentlitteratur, 1999.

[6] HAMMOND, P.: Applied Electromagnetism. Pergamon Press : Oxford, 1985.

[7] JIN, J.: The Finite Element Method in Electromagnetics, vol. 2, John Wiley \& Sons, 2002.

[8] DODD, C. V., DEEDS, W. E.: Analytical Solutions to Eddy-current Probecoil Problems. J. of Applied Physics, 39(6), 2829-2838, 1968.

[9] CHABOUdEZ, C., ClAIN, S., GLARDON, R., MARI, D., RAPPAZ, J., SWIERKOSZ, M.: Numerical Modeling in Induction Heating for Axisymmetric Geometries. Magnetics, IEEE Transactions, 33(1), 739-745, 1997.

[10] DAVIES, E. J.: Conduction and Induction Heating. No. 0-86341-174-6 in IEE Power Engineering Series 11. Peter Peregrinus Ltd. : London, 1990.

[11] MATTEW, N., SADIKU, O.: Elements of Electromagnetics, vol. 3, Oxford University Press, 2001.

[12] WANG, K. F., CHANDRASEKAR, S., HENRY, T., YANG, Y.: Finite-element Simulation of Induction Heat Treatment. J. of Materials Engineering and Performance, 1, 1992.

[13] CARSLAW, H. S., JAEGER, J. C.: Conduction of Heat in Solids, $2^{\text {nd }}$ ed., Oxford University Press : Oxford, 1959.

[14] CALLISTER, JR. W. D.: Materials Science and Engineering: An Introduction. John Wiley \& Sons, 1999.

[15] LEWIS, R. W., MORGAN, K., THOMAS, H. R., SEETHARAMU, K. N.: The Finite Element Method in Heat Transfer Analysis. John Wiley \& Sons, 1996.

[16] LEWIS, R. W., NITIARASU, P., SEETHARAMU, K. N.: Fundamentals of the Finite Element Method for Heat and Fluid Flow. Wiley : Chichester, 2004.

[17] LABRIDIS, D., DOKOPOULO, P.: Calculation of Eddy Current Losses in Nonlinear Ferromagnetic Materials. IEEE Transactions on Magnetics, 25(3), May 1989.

[18] JAKUBOVICOVA, L., KOPAS, P., HANDRIK, M., VASKO, M.: Computational and Experimental Analysis of Torsion and Bending Loading of Specimen. In-Tech 2010, September 2010, Prague, pp. 395-400.

[19] ZMINDAK, M., NOVAK, P.: Particles Interactions in Composites Reinforced by Fibre and Spherical Inclusions. Communications - Scientific Letters of the University of Zilina, vol. 11, No. 2, pp. 13-18, 2009.

[20] SAGA, M., VASKO, M.: Stress Sensitivity Analysis of the Beam and Shell Finite Elements. Communications - Scientific Letters of the University of Zilina, vol. 11, No. 2, 2009, pp. 5-12.

[21] KWON, Y. W., BANG, H.: The Finite Element Method using MATLAB. CRC Press : University of Minnesota, 1996.

[22] SAPIETOVA, A., DEKYS, V., VASKO, M.: A Numerical Modeling Rotating Machine Having Unbalance and the Measuring of its Dynamical Properties, Metalurgija/Metallurgy, vol. 492010, No. 2, pp. 503-507. 